\title{
Interim Analysis of Phase 2 Results for Cemiplimab in Patients with Metastatic Basal Cell Garcinoma (mBCC) who Progressed on or are Intolerant to Hedgehog Inhibitors (HHIs)
}

Karl D. Lewis, ${ }^{1}$ Ketty Peris, ${ }^{2}$ Aleksandar Sekulic, ${ }^{3}$ Alexander J. Stratigos, ${ }^{4}$ Lara Dunn, ${ }^{5}$ Zeynep Eroglu, ${ }^{\circ}$ Anne Lynn S. Chang,,${ }^{7}$ Michael R. Migden, ${ }^{8}$ Siyu Li, ${ }^{9}$ Suk-Young Yoo, ${ }^{9}$ Kosalai Mohan, ${ }^{10}$ Ebony Coates, ${ }^{10}$ Emmanuel Okoye, ${ }^{10}$ Jean-François Baurain, ${ }^{11}$ Oliver Bechter, ${ }^{12}$ Axel Hauschild, ${ }^{13}$ Marcus O. Butler, ${ }^{14}$ Leonel Hernandez-Aya,,${ }^{15}$ Lisa Licitra,,${ }^{16}$ Rogerio I. Neves, ${ }^{17}$ Emily S. Ruiz,,${ }^{18}$ Frank Seebach, ${ }^{10}$ Israel Lowy, ${ }^{10}$ Timothy Bowler, ${ }^{10}$ Matthew G. Fury

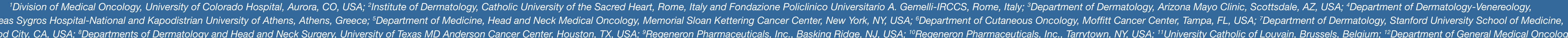

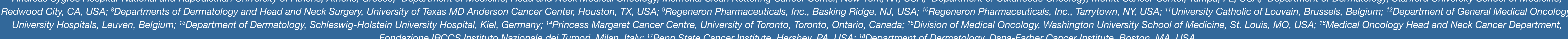

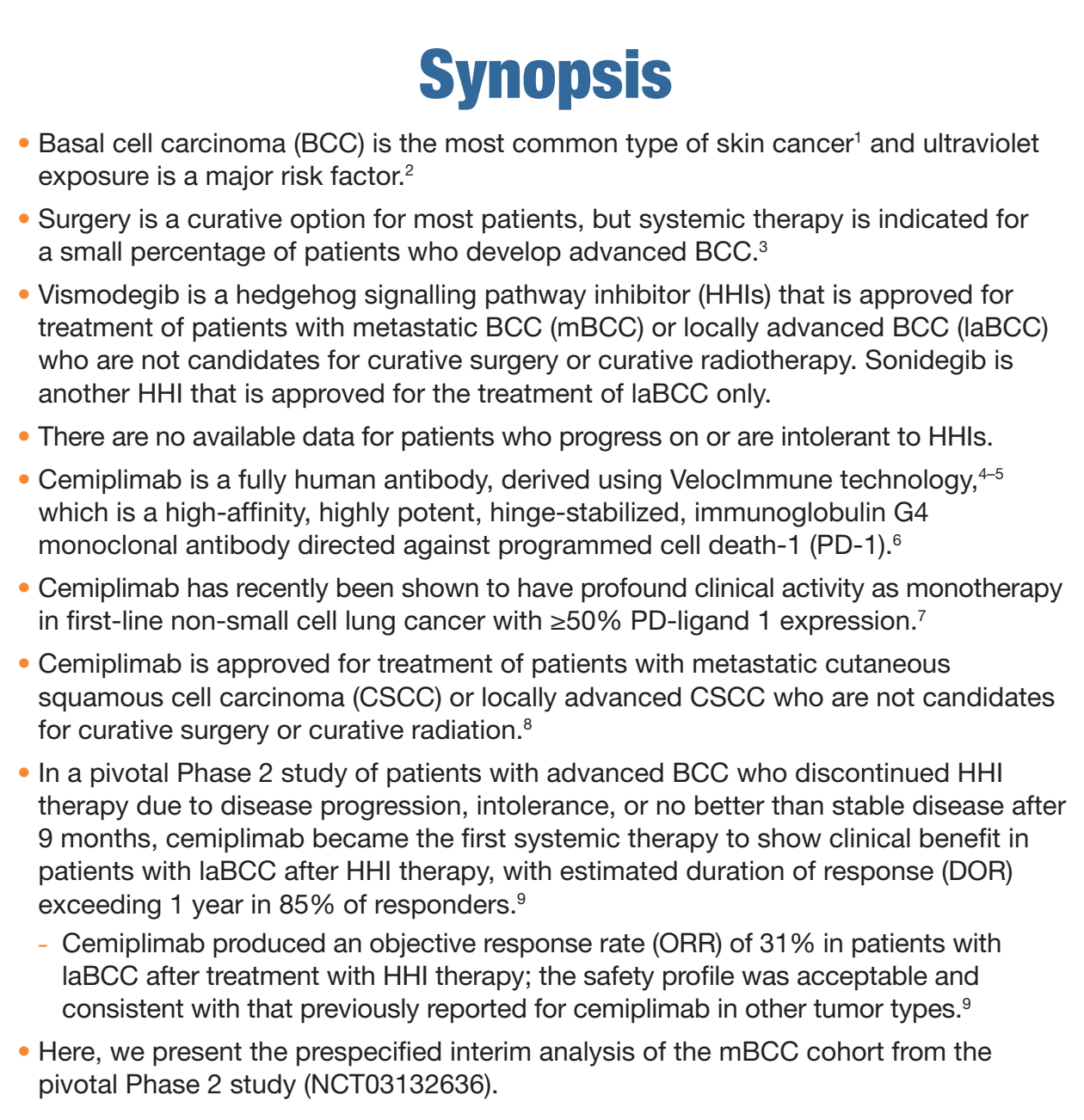

Objectives

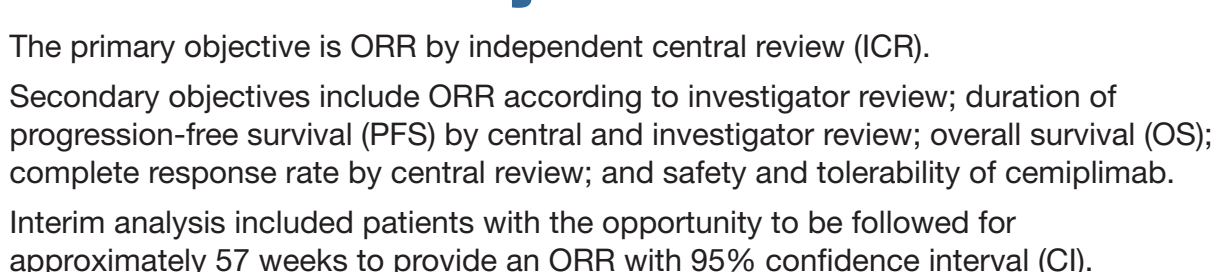

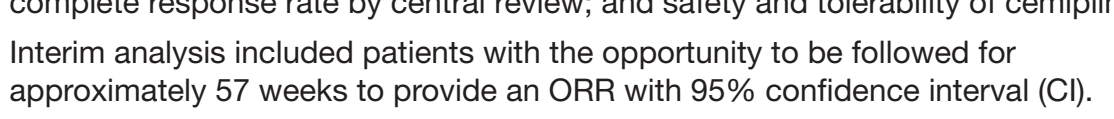

Methods

This is a Phase 2, non-randomized, multi-center study of cemipilimab in patients with
efther mBCC or abCC (Figure 1).

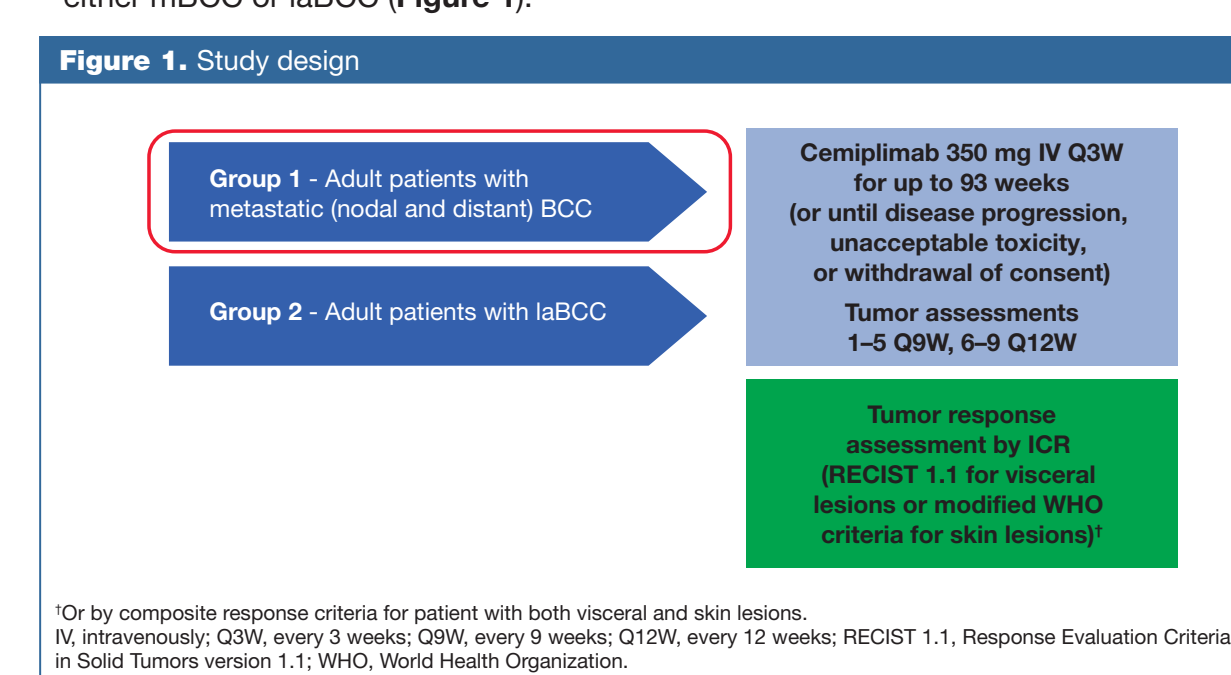

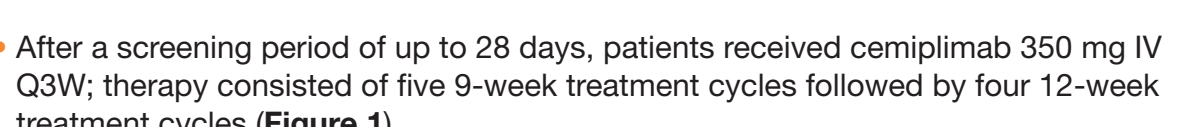

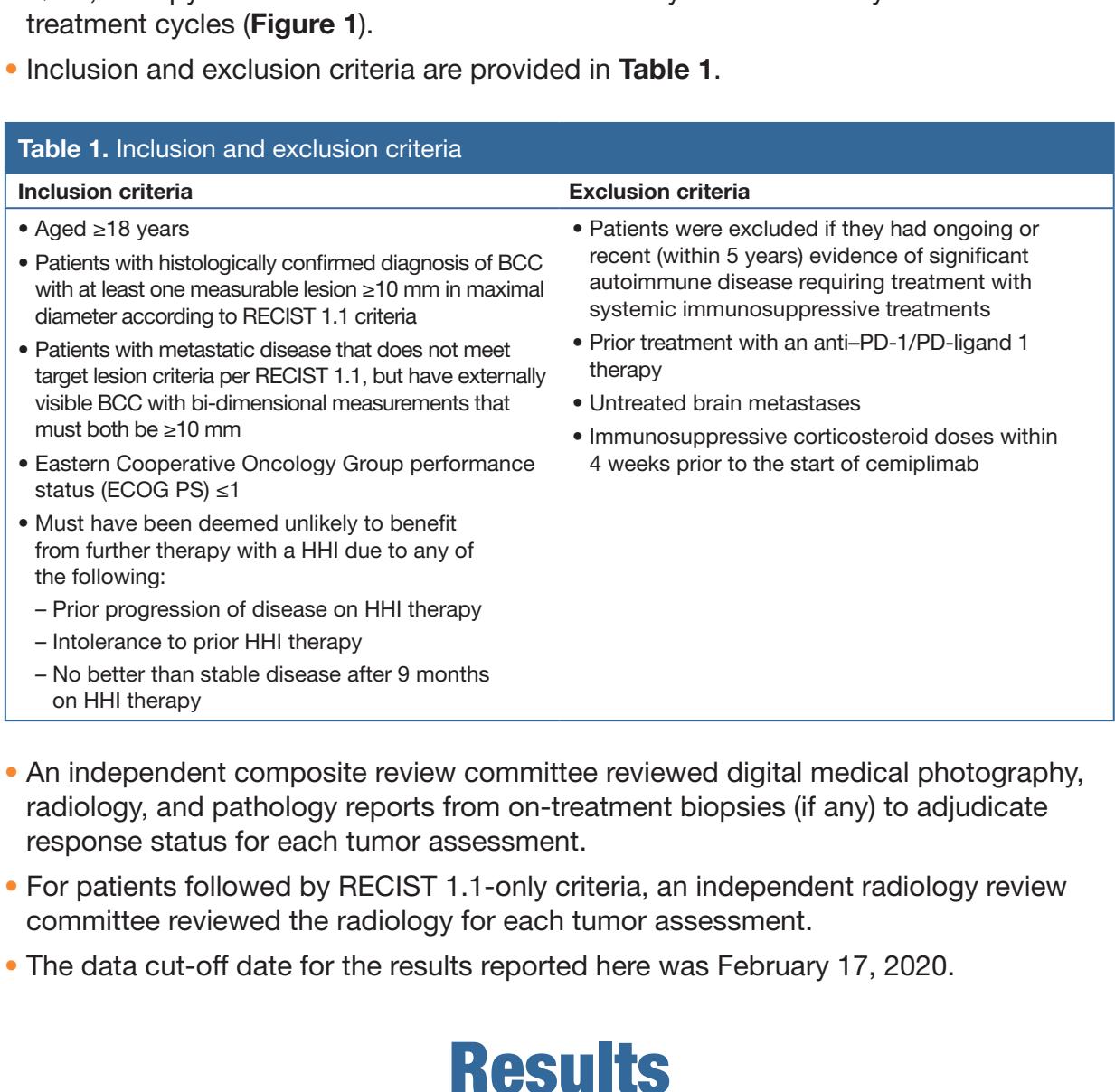

Patients
- As of datat cut-off, 28 patients with mBCC had sufficient follow-up to be considered
evaluable tor colinical activty; $82.1 \%$ were males and median age was 65.5 years

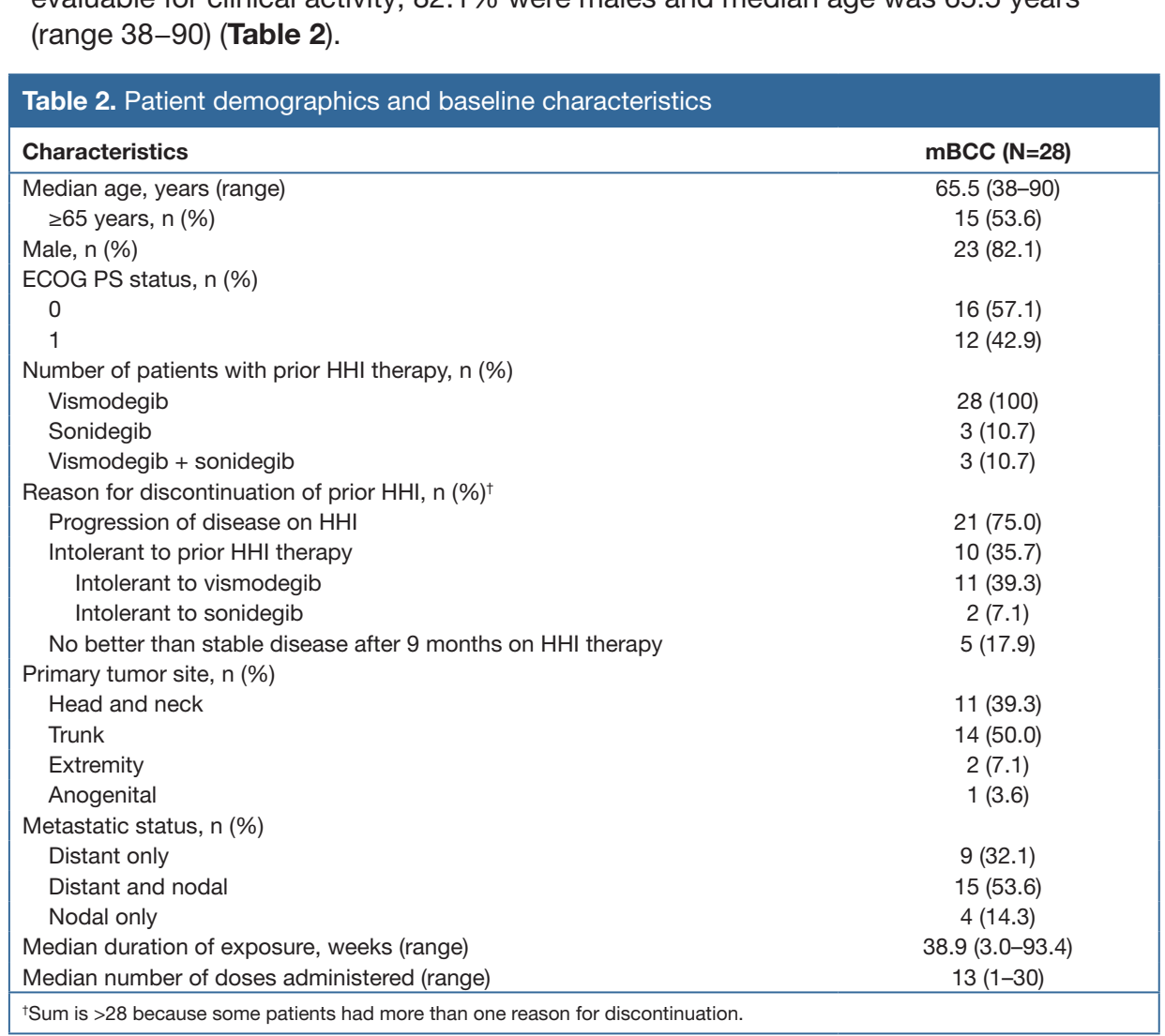

Clinical activity
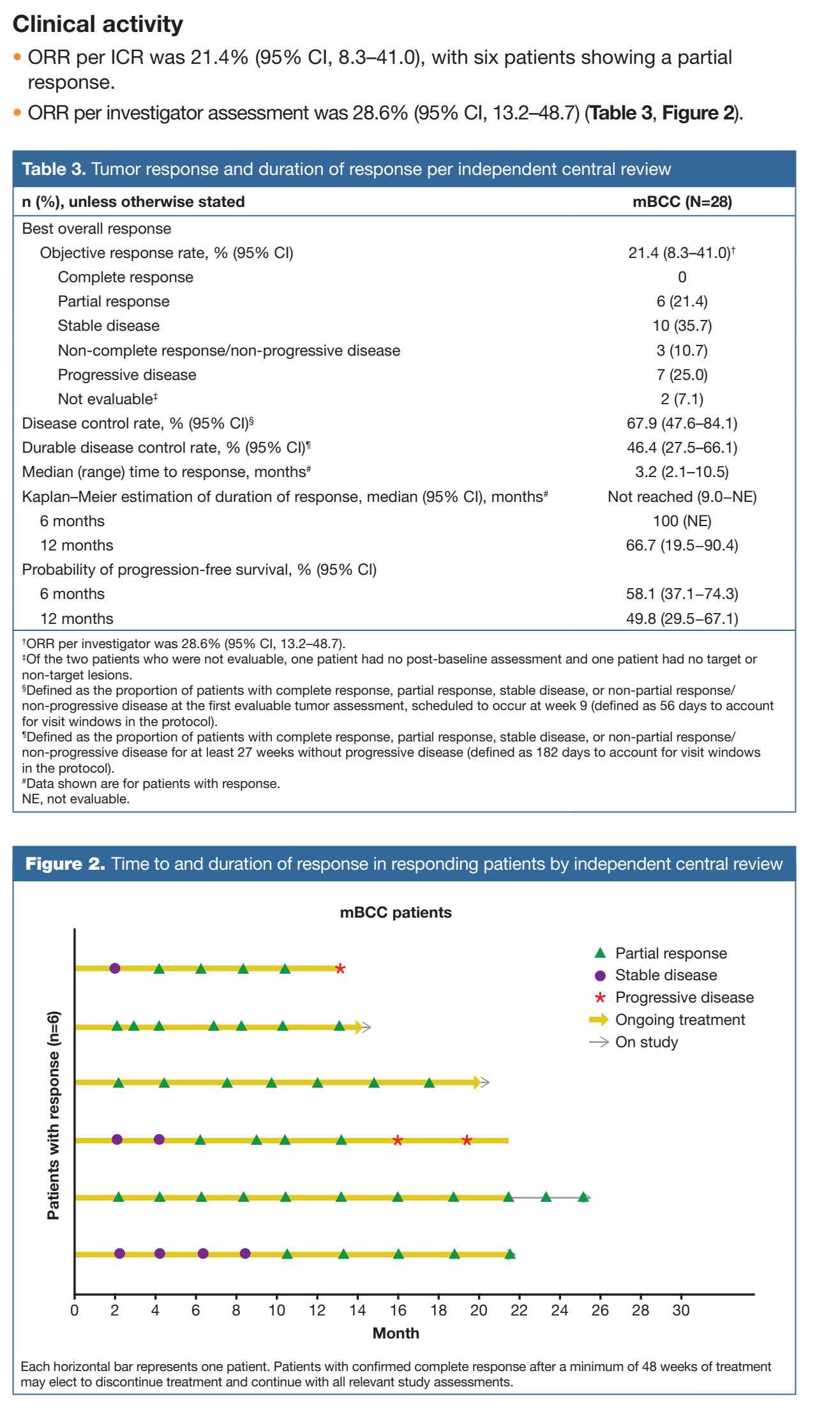

The disease control rate was $67.9 \%$ (99\%) C1, 47.6-684.1).

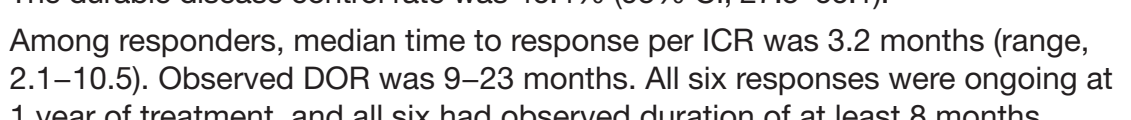

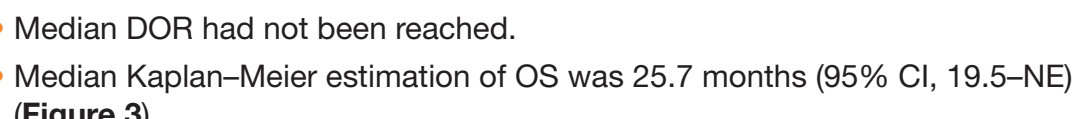

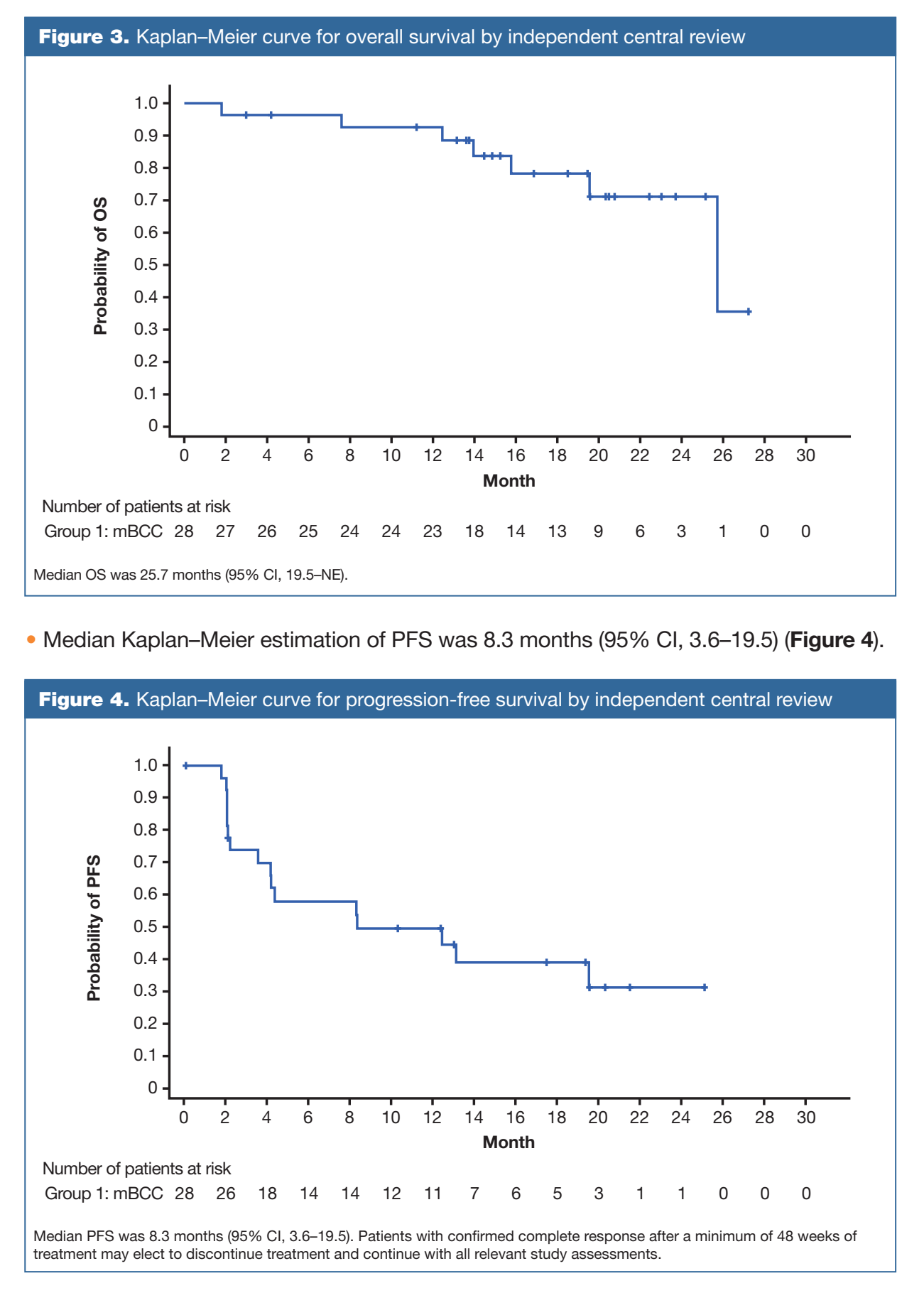

Safety

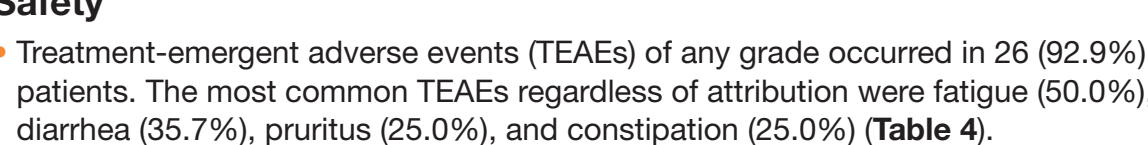

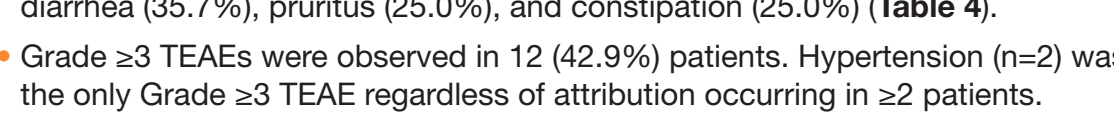

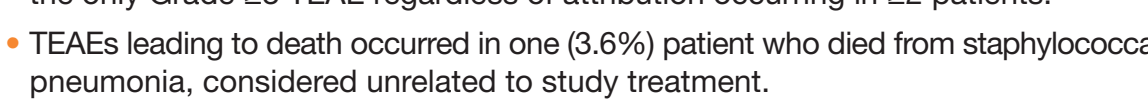

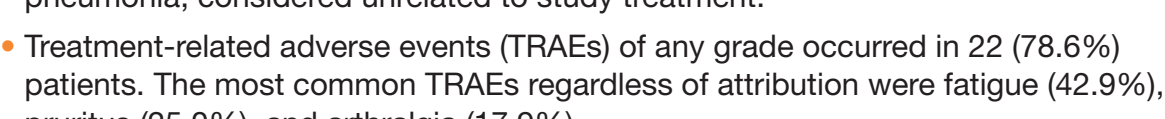
- Grade 23. TRAAs were observed in five (17.9\%) patients

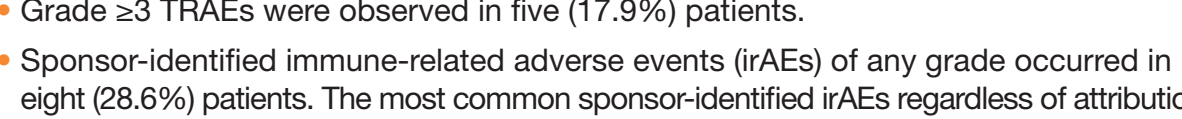
were autoimmune hepatitis, colitis, hypothyrodidism, and pneumonitis (each h.7.1\%).

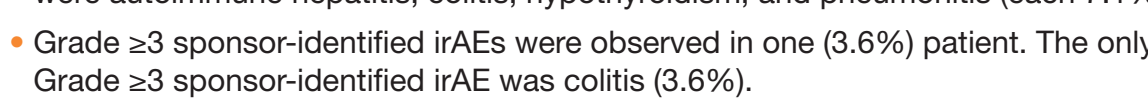

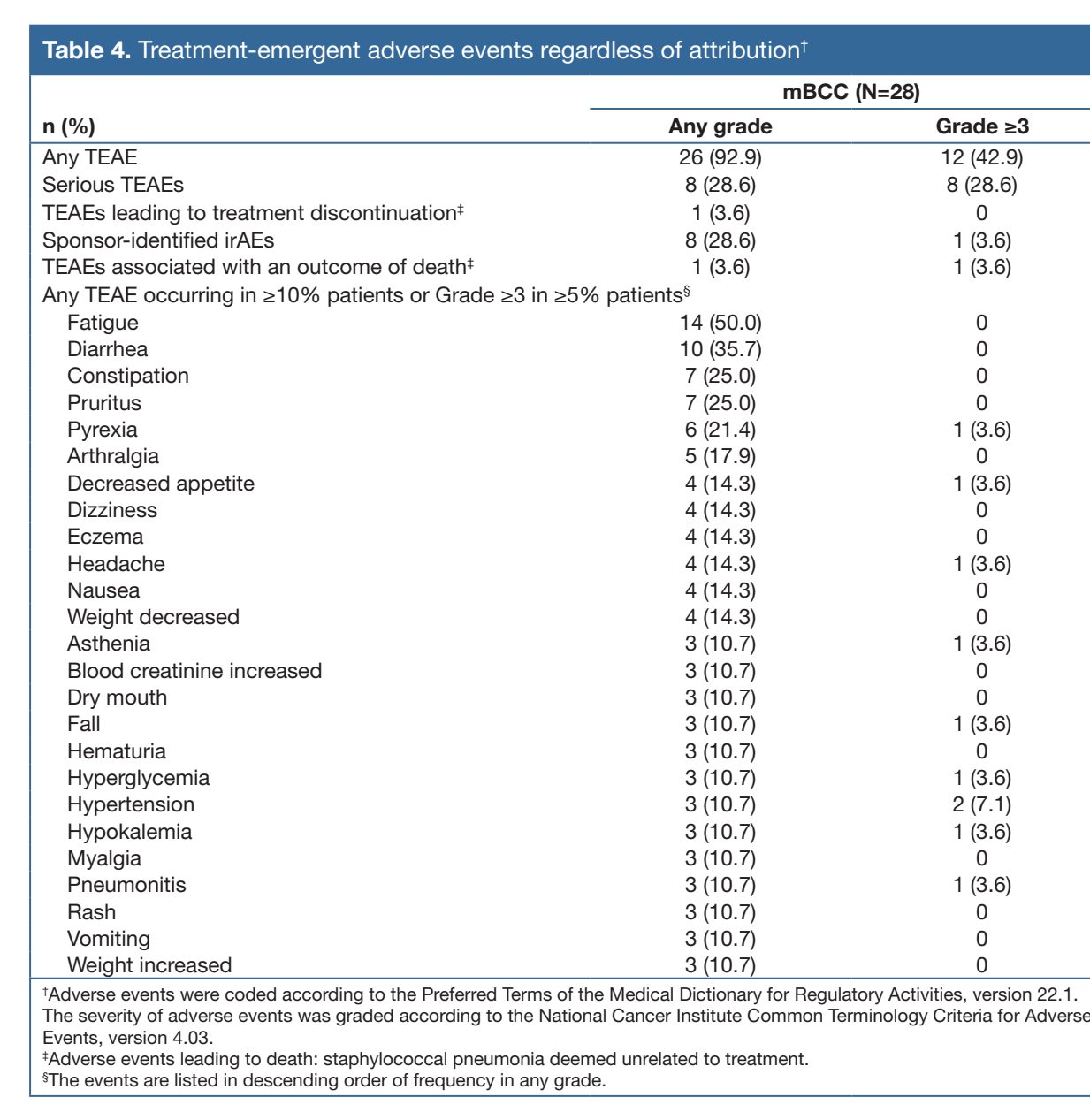

Summary and Conclusions - This interim analysis demonstrates that cemilinabab is the first agent to provide
clinically meaningulu antitumar activity, including durable responses, in patients The safety profile of cemiplinab is consistent with previous reports of cemipilimab in other tumor types.
- Combined with data trom the laBCC conorot, these results confirm that cemiplimab
has substantial activity in advanced BCC tumors.

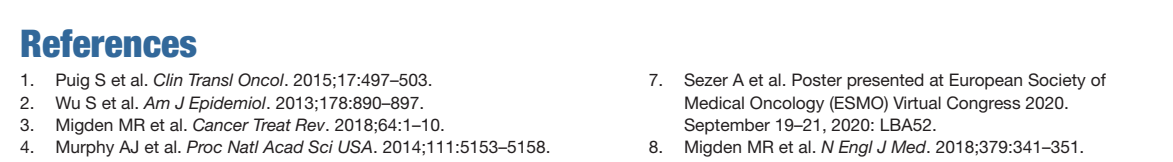

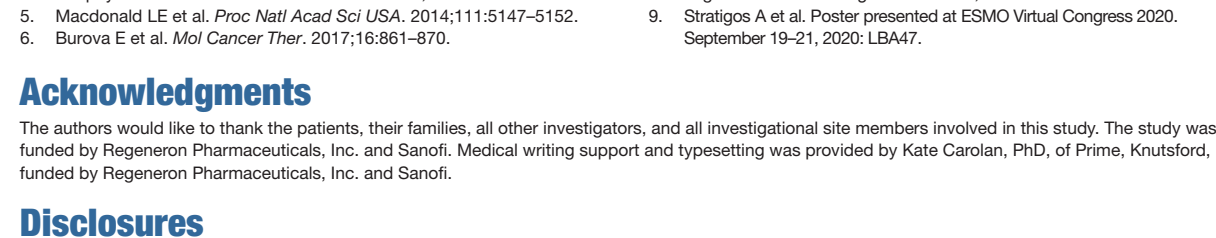

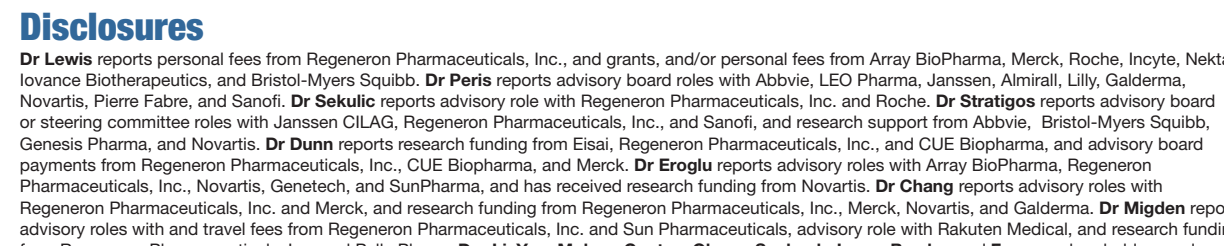

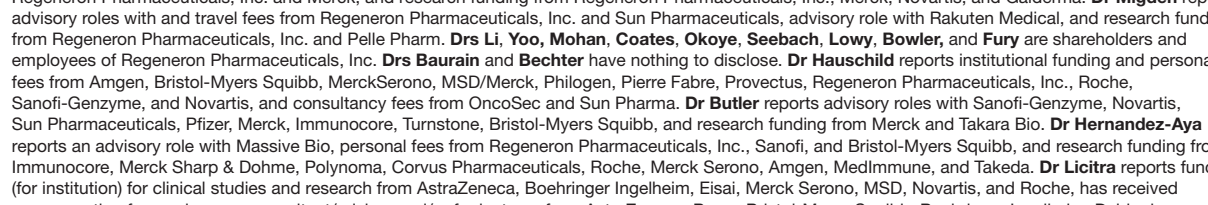

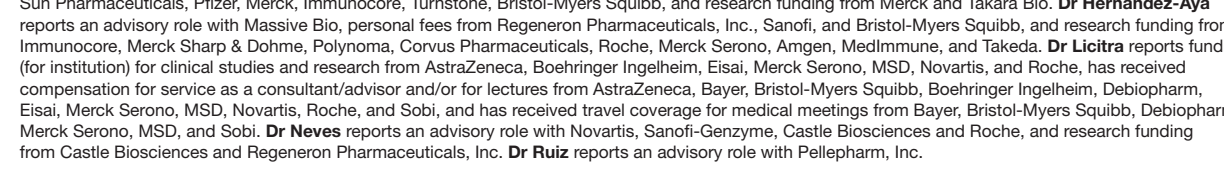

